\title{
FRACTIONAL VISCOSITY DEPENDENCE OF RELAXATION RATES AND NON-STEADY-STATE DYNAMICS IN BARRIERLESS REACTIONS IN SOLUTION
}

\author{
Biman BAGCHI \\ Solid State and Structural Chemistry Unit, Indian Institute of Science, Bangalore, 560012 , India
}

Received 13 March 1987; in final form 8 May 1987

\begin{abstract}
A finite decay model of barrierless electronic relaxation in solution is studied both analytically and numerically. The fractional viscosity $(\eta)$ dependence of the rate $\left(k \propto \eta^{-\alpha}\right.$ with $\left.1>\alpha>0\right)$ is shown to be strongly correlated with non-steady-state dynamics on the reaction potential surface. We also find that the exponent $\alpha$ may depend strongly on the wavelength of the exciting light.
\end{abstract}

\section{Introduction}

The viscosity dependence of the non-radiative relaxation rate in barrierless electronic relaxation in solution has been a subject of interest for many years [1-20]. In many cases, the decay rate can be fitted fairly well to an expression of the following form $[1,4-7,10,14,15]$ :

$k=C \eta^{-\alpha}$,

where the exponent $\alpha$ is usually less than unity, $1>\alpha>0$ and $C$ is a viscosity-independent constant. Although a quantitative understanding of the origin of (1) is still lacking, one can identify several plausible explanations for a dependence of the kind given by eq. (1). Firstly, competition between radiative and non-radiative relaxation may lead to a weak viscosity dependence at large viscosities. This is because radiative decay is mostly position-independent whereas certain critical geometries must be attained for non-radiative relaxation to occur. Thus, at small viscosity the electronic relaxation rate may depend inversely on viscosity $(\alpha=1)$ but at large viscosities a cross-over to (1) with $\alpha$ less than unity may take place. Secondly, the solvent frictional forces on the reactive motion may not be related to the macroscopic viscosity by the linear Stokes-Einstein relation. This breakdown of the Stokes-Einstein relation has been a subject of several recent discussions $[21,22]$. Thirdly, the reaction potential surface itself may change significantly as the viscosity of the solvent is changed [12]. This is plausible because it is seldom possible to change the viscosity of a polar solvent without changing the diclectric constant of the medium. And fourthly, the fractional viscosity dependence may arise as an intrinsic property of the barrierless reaction. It is the fourth point that is the subject of this Letter. Note that by careful experimentation, it may be possible to eliminate the first three possibilities and study the rate that is intrinsic to a particular reaction.

In order to study the dynamics of barrierless reactions, we introduce a new form of the sink function in this Letter. We shall assume that the position dependence of the sink is given by a delta function positioned at the origin of a harmonic surface. The time evolution of the excited-state population density, $P(x, t)$, is given by the following modified Smoluchowski equation $[14,15]$,

$\frac{\partial P}{\partial t}=A \frac{\partial^{2} P}{\partial x^{2}}+B \frac{\partial}{\partial x} x P-k_{0} S(x) P$,

where $A=k_{\mathrm{B}} T / \zeta, B=\mu \omega^{2} / \zeta, \zeta$ being the relevant friction parameter, $\omega$ the frequency of the (assumed) harmonic surface, $\mu$ the effective mass, $T$ the temperature and $k_{\mathrm{B}}$ is the Boltzmann constant. $S(x)$ is the sink function $[14,15]$ and $k_{0}$ is the rate of nonradiative decay from the sink. In writing (2) we have neglected the contribution from radiative decay as we are interested only in the dynamics of non-radia- 
tive relaxation. The sink function $S(x)$ is assumed to be a delta function located at the origin of the harmonic surface

$S(x)=\delta(x)$

The motivation for introducing the delta function sink is twofold. Firstly, it simplifies the numerical calculations substantially and at the same time retains the essential features of the more general (and realistic) Gaussian sink function $[14,15]$. The deltafunction sink is obtained from the Gaussian sink function by taking the limit that the width of the sink is infinitesimally small. Secondly, for many realistic situations, (3) is a reasonable approximation [13].

In a previous study [15], eq. (2) was solved numerically for a Gaussian sink. However, in that study specific values of $k_{0}$ and other parameters were used and as a result some general features of the relaxation were not recognised. In the present study, we shall transform eq. (2) to a form in which the role of several competing processes will become transparent. This will allow us to make several general conclusions about barrierless chemical reactions. We find that the non-steady-state dynamics on the excited-state potential surface gives rise to a viscosity dependence of the form (1), especially for the time-integrated rate constant, $k_{\mathrm{I}}$. Another interesting finding of this study is that the exponent $\alpha$ may depend rather strongly on the excitation wavelength in the intermediate viscosity range. Multi-exponential decay of the excited-state population is shown to be a direct consequence of this non-steady-state dynamics.

At this point it is necessary to discuss briefly the nomenclature adopted here. We shall call the models described by eq. (2) with finite $k_{0}$ as the finite decay models. These models are significantly different from the instantaneous death models where the excited state decays with unit probability as certain critical geometries (funnels or holes) are reached. Examples of the latter class of models are the pinhole sink model $[14,15]$, the model of Oster and Nishijima [2] and the staircase model introduced recently by the present author [18]. These models predict an inverse viscosity dependence of the rate at all viscosities. Also note that these models can be obtained from (2) by taking the limit $k_{0} \rightarrow \infty$. The main advantage of the instantaneous death models is that they can be solved analytically.

In section 2 we present the theoretical results. In section 3 the numerical results and discussion are given.

\section{Theory}

We first note that eq. (2) contains three independent time constants, $k_{0}^{-1}, B^{-1}$ and $A^{-1}$ (when the reactive motion is rotational diffusion; for translational motion it would be $a^{2} / A$ with $a$ some appropriate length scale). In particular the long time decay is controlled by $k_{0}$ and $B$. It is thus convenient to rewrite eq. (2) in the following form:

$\frac{\partial P}{\partial t_{1}}=\tilde{A} \frac{\partial^{2} P}{\partial x^{2}}+\frac{\partial}{\partial x} x P-\tilde{k}_{0} S(x) P$,

where

$t_{1}=B t$

and

$\tilde{A}=A / B, \quad \tilde{k}_{0}=k_{0} / B$

The dimensionless quantity $\tilde{k}_{0}$ is the most important quantity in the dynamical process. Another advantage of (4) is that $\tilde{A}$ is independent of viscosity. We now show that from (4) one can obtain simple limiting expressions for the long-time rate constant $k_{\mathrm{L}}$. $k_{\mathrm{L}}$ is defined by

$k_{\mathrm{L}}=-\lim _{t \rightarrow \infty} \frac{\partial}{\partial t} \ln P_{\mathrm{e}}(t)$,

where $P_{\mathrm{c}}(t)$ is the total, time-dependent, population on the excited-state surface at time $t$. Because $\tilde{A}$ is independent of $\zeta$ and because of the form of eq. (4), we can write $\tilde{k}_{\mathrm{L}}$ as

$\tilde{k}_{\mathrm{L}}=f\left(\tilde{k}_{0}\right)$,

where $f$ is a function whose limiting forms are given below. From (6), we get

$k_{\mathrm{L}}=B f\left(\tilde{k}_{0}\right)$.

In the limit $\tilde{k}_{0} \rightarrow \infty$, we recover the result of the pinhole sink model, i.e. $f \rightarrow 1$. In this limit an inverse vis- 
cosity dependence is predicted. In the limit of small $k_{0}$, the long time decay will be dominated by $k_{0}$ and $f\left(\tilde{k}_{0}\right) \sim \tilde{k}_{0}$ so $k_{\mathrm{L}} \sim k_{0}$. In this limit, $k_{\mathrm{L}}$ is independent of viscosity. One can thus expect a fractional viscosity dependence for intermediate values of $\tilde{k}_{0}$.

If the decay is multi exponential then the more useful quantity is the integrated rate constant, $k_{\mathrm{I}}$, defined by

$k_{\mathrm{I}}^{-1}=\int_{0}^{\infty} \mathrm{d} t P_{\mathrm{e}}(t)$.

We shall show below that $k_{\mathrm{I}}$ has a more interesting viscosity dependence than $k_{\mathrm{L}}$.

Next, we proceed to solve eq. (4) numerically. We expand $P(x, t)$ in the eigenfunctions of the associated Fokker-Planck (FP) operator

$P(x, t)=\sum_{n=0}^{\infty} b_{n}(x) \sigma_{n}(t)$,

where $b_{n}$ is the $n$th eigenfunction of the FP operator [15] given by

$b_{n}(x)=A_{n}^{1 / 2} \exp \left(-x^{2} / 2 V^{2}\right) H_{n}\left(x / 2^{1 / 2} V\right)$,

where $H_{n}$ is the Hermite polynomial of order $n$, $V=\tilde{A}^{1 / 2}$ and the normalization constant, $A_{n}$, is given by

$A_{n}=\left[V n ! 2^{n}(2 \pi)^{1 / 2}\right]^{-1}$.

By using standard procedures we obtain the following system of equations for $\sigma_{n}(t)$ :

$\dot{\sigma}_{m}\left(t_{1}\right)=-m \sigma_{m}\left(t_{1}\right)-\tilde{k}_{0} \sum J_{m n} \sigma_{n}\left(t_{1}\right)$

with

$J_{m n}=A_{m}^{1 / 2} A_{n}^{1 / 2} H_{m}(0) H_{n}(0)$.

$P_{\mathrm{c}}(t)$ is given by [15]

$P_{\mathrm{e}}\left(t_{\mathrm{l}}\right)=\left[(2 \pi)^{1 / 2} V\right]^{1 / 2} \sigma_{0}\left(t_{1}\right)$

and the expression for $k_{\mathrm{I}}$ is

$k_{\mathrm{I}}^{-1}=\left[(2 \pi)^{1 / 2} V\right]^{1 / 2} \hat{\sigma}_{0}(s=0)$,

where $\hat{\sigma}_{0}(s)$ is the Laplace transform of $\sigma_{0}(t)$

$\hat{\sigma}_{0}(s)=\int_{0}^{\infty} \mathrm{d} t \exp (-s t) \sigma_{0}(t)$.
In the preceding discussion we have set $B=1$, so $t$ and $k_{\mathrm{I}}^{-1}$ are in units of $B$. Since $J$ is a symmetric matrix, it is straightforward to diagonalize the matrix $\left(m \mathbf{l}+k_{0} \mathrm{~J}\right)$ to obtain the eigenvalues $\lambda$. The lowest eigenvalue $\lambda_{1}$ gives the long-time rate constant $k_{\mathrm{L}}$. For calculation of $k_{\mathrm{l}}$, we found it more convenient to use eq. (15) and obtain $\hat{\sigma}_{0}(s=0)$ directly by matrix inversion. The initial condition $P(x, t=0)$ $=x-x_{0}$ gives

$\sigma_{m}(0)=b_{m}^{\dagger}\left(x_{0}\right)$.

It is straightforward to generalize (17) for a realistic initial distribution $[18,23]$ which primarily affects the short time dynamics but leaves the main conclusions presented here unchanged.

\section{Results and discussion}

In this section we present the results of our numerical solution of the system of equations (12) and (13) with the initial conditions given by (17). At smaller values of $\tilde{k}_{0}\left(\tilde{k}_{0} \lesssim 1\right)$, good convergence is obtained with a small number (20-30) of even eigenstates. For larger $\tilde{k}_{0}\left(2 \leqslant \bar{k}_{0} \leqslant 50\right)$, a larger number $(60-100)$ of even eigenstates is necessary to obtain satisfactory convergence. Both the eigenvalue spectrum and the integrated rate constant, $\tilde{k}_{1}$, were obtained numerically on a DEC-1090 computer. The results are presented below.

\subsection{Non-steady-state dynamics and multi-exponential decay}

The condition for a single exponential decay is that the lowest eigenvalue, $\lambda_{1}$, should be substantially smaller than the next highest eigenvalue, $\lambda_{2}$. Thus a suitable parameter to study multi-exponential decay is

$\Delta_{12}=\left(\lambda_{2}-\lambda_{1}\right) / \lambda_{1}$.

For single exponential decay to dominate the overall relaxation process, $\Delta_{12}$ must be much greater than unity. This is also the condition for a steady (constant) rate of decay from the sink region. 


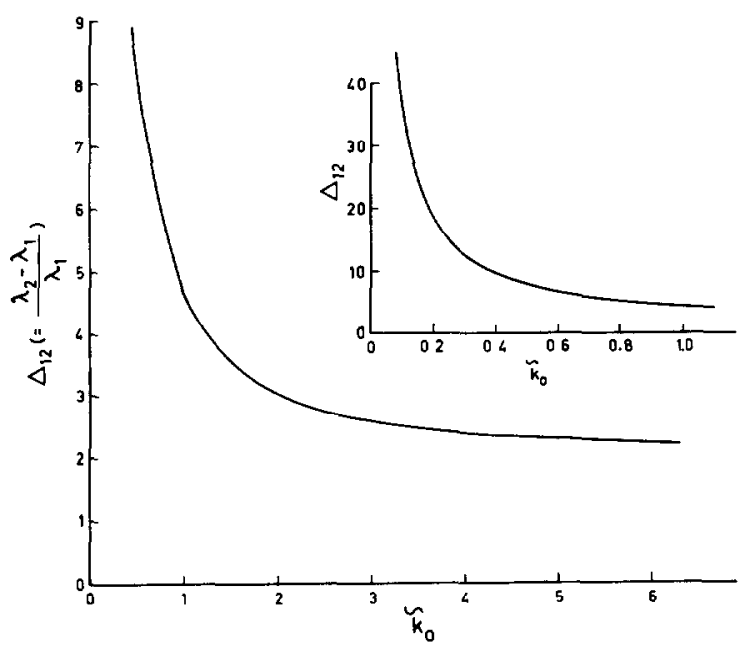

Fig. 1. The calculated value of $\Delta_{12}$ plotted against $\tilde{k}_{0}\left(=k_{0} / B\right)$. The inset shows the behaviour for small values of $\tilde{k}_{0}$ on an expanded scale.

In fig. 1 we have plotted the values of $\Delta_{12}$ against $\tilde{k}_{0}\left(=k_{0} / B\right)$. The inset in this figure is for very small values of $\tilde{k}_{0}$. It is seen clearly that $\Delta_{12}$ becomes very large at small values of $\tilde{k}_{0}$ indicating a single exponential behaviour. At larger values of $\tilde{k}_{0}\left(k_{0} \gtrsim\right.$ $2), \Delta_{12}$ becomes smaller and comparable to unity. It is in this range that multi-exponential behaviour begins to dominate the relaxation process. In fig. 2 we have plotted the time-dependent excited state population, $P_{\mathrm{c}}(t)$, against time for three values of $\bar{k}_{0}$. The increasing non-exponential character of the

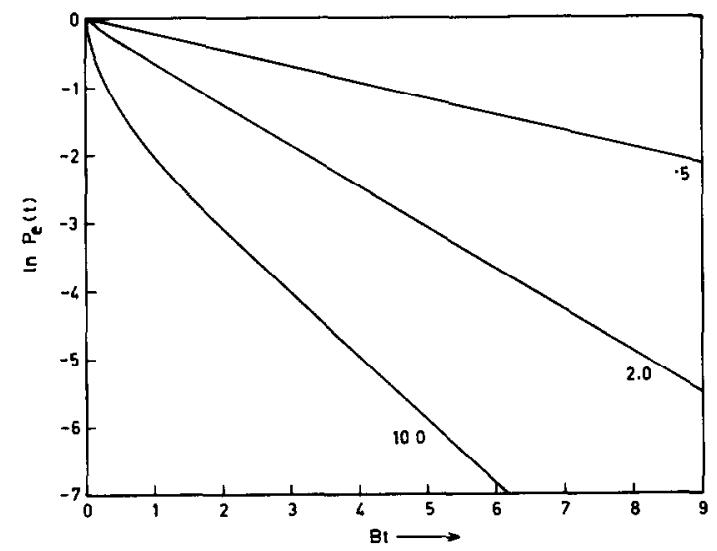

Fig. 2. The excited-state population $P_{\mathrm{e}}(t)$ at time $B t$ plotted against $B t$ for several values of the dimensionless quantity $\tilde{k}_{0}$. The values of $\tilde{k}_{0}$ are given in the figures. $x_{0}=0.5$. decay, as $\tilde{k}_{0}$ is increased, is clear from the graphs.

Since the results are given in terms of the dimensionless quantity $\tilde{k}_{0}$, there are two ways to interpret them. Firstly, we can keep $B$ (i.e. the friction) constant and vary $k_{0}$. Alternatively, we can fix $k_{0}$, and change $B$. In the latter case, figs. 1 and 2 depict the effects of changing the viscosity on the relaxation process. As friction increases, $B$ decreases and $\tilde{k}_{0}$ increases. So, low $\tilde{k}_{0}$ corresponds to low friction and large $\tilde{k}_{0}$ to large friction. Thus fig. 2 shows the crossover to non-exponential behaviour as $\zeta$ increases. This has been observed experimentally in some cases [6-9] and discussed theoretically in our earlier paper [15]. Note that the scale changes in fig. 2 as $B$ is changed. For larger $\widetilde{k}_{0}, B$ is also larger. At large values of $B t(B t \gg 1)$, the relaxation becomes single exponential for all $\tilde{k}_{0}$. However, the signal in this region may become too low in some cases to detect this long-time single exponential behaviour.

\subsection{Non-steady-state dynamics and fractional viscosity dependence of rate}

We present two important results here. The first is concerned with the significantly different viscosity dependence of the two rate constants, $k_{\mathrm{L}}$ and $k_{\mathrm{l}}$. Secondly, we find that, for $k_{\mathrm{I}}$, the exponent $\alpha$ of eq. (1) depends on the excitation wavelength.

In fig. 3 we have plotted both the lowest eigenvalue $\tilde{\lambda}_{1}\left(=\tilde{k}_{\mathrm{L}}\right)$ and the integrated rate constant $\tilde{k}_{\mathrm{I}}$

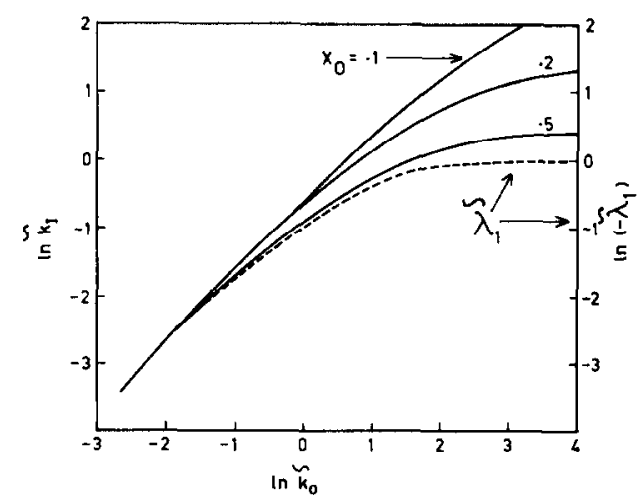

Fig. 3. Values of $\tilde{\lambda}_{1}$ and $\tilde{k}_{1}$ plotted against $\tilde{k}_{0} . \tilde{k}_{\mathrm{l}}$ is plotted for several values of the initial position $x_{0}$ on the excited state potential surface. The values of $x_{0}$ are indicated on the graph. 
against $\tilde{k}_{0}$. The values of $\tilde{k}_{1}$ are given for several values of the initial position $x_{0}$. As $\tilde{k}_{0}$ becomes larger, $\tilde{\lambda}_{1}$ approaches unity which means $\lambda_{1}$, and $k_{\mathrm{L}}$, becomes equal to $B$. This is of course expected from the known result [15] for the pinhole sink. Thus $k_{\mathrm{L}}$ has a viscosity dependence of the form given by eq. (1) (with exponent $\alpha$ less than unity) only for high values of $B$. Thus, at fixed $k_{0}$, a fractional viscosity dependence of $k_{\mathrm{L}}$ is predicted only at small viscosities, in agreement with the theoretical analysis presented earlier in this section.

However, the behaviour of $k_{\mathrm{I}}$ is significantly different from that of $k_{\mathrm{L}}$. Firstly, the values of $\tilde{k}_{\mathrm{I}}$ seem to saturate at larger values of $\tilde{k}_{0}$ which means fractional viscosity dependence will be observed over a larger range of viscosity. Secondly, the variation of $\tilde{k}_{1}$ with $\tilde{k}_{0}$ depends strongly on the initial conditions. For example, for $x_{0}=0.1$, fractional viscosity dependence is predicted even beyond $\tilde{k}_{0} \gtrsim 50$. This is in the range available to experimental studies. For TPM dyes, $B \approx 10^{10}-10^{11} \mathrm{~s}^{-1}$ at $\eta=1 \mathrm{cP}$ if the reactive motion is a synchronous rotation of the phenyl rings, and $k_{0} \approx 10^{12} \mathrm{~s}^{-1}$. However, $k_{0}$ may also change with viscosity because a change in viscosity is usually accompanied by a change of the dielectric properties of the solvent. Thus, although we predict a fractional viscosity dependence of $k_{\mathrm{l}}$, it is not possible, within this theory, to attribute it solely to viscous effects.

The preceding discussion suggests that experimental analysis of the viscosity dependence should be carried out by obtaining both $k_{\mathrm{J}}$ and $k_{\mathrm{L}}$ (if single exponential decay is attained at long times) as functions of viscosity at various excitation wavelengths in similar (with regard to polar properties) solvents. At the viscosity usually attained in experiments and for the type of molecules that undergo barrierless reaction via large amplitude motion, the long-time decay rate $k_{L}$ will probably show inverse viscosity dependence at all viscosities. But $k_{\mathrm{I}}$ may show a fractional viscosity dependence at small to intermediate viscosity values. Moreover, $k_{\mathrm{I}}$ may show a dependence on the excitation wavelength but $k_{\mathrm{L}}$ should be independent of it.

We would like to stress here that if an instantaneous death model is appropriate for a given situation, then an inverse viscosity dependence would be observed at all viscosities for both $k_{\mathrm{L}}$ and $k_{\mathrm{I}}$. Thus careful experimental studies are necessary to understand all these competing factors.

\subsection{Temperature dependence of rate}

At low values of $\tilde{k}_{0}$ an equilibrium (Boltzmann) distribution is attained on the excited state surface before any significant decay can take place. Increase of the temperature then widens the Boltzmann distribution and removes population from the sink region, giving rise to a decrease in the rate of decay. Thus, we would have an apparent "negative" activation energy at low values of $\tilde{k}_{0}$. But as $\tilde{k}_{0}$ is increased, the Boltzmann distribution is never attained, the rate determining step is now the time the system takes to reach the sink and the rate increases with $T$. Thus, a cross-over to positive activation energy is predicted. However, note that $k_{0}$ may itself increase with $T$, thus giving rise to an activation energy larger than that expected from the viscous effects alone.

We have the following concluding remarks. The treatment presented here is one dimensional and needs to be generalized. The major differences between instantaneous death models (such as the pinhole sink) and finite decay models (such as the delta-function sink) are expected to persist in the higher dimensions. Finally, the dependence of relaxation on the wavelength of the exciting light deserves to be fully explored in experiments. Much valuable information on barrierless reaction may be gained from such studies.

\section{References}

[1] Th. Förster and G. Hoffmann, Z. Physik 75 (1971) 63.

[2] G. Oster and Y. Nishijima, J. Am. Chem. Soc. 78 (1956) 1581.

[3] W. Rapp. Chem. Phys. Letters 27 (1974) 187.

[4] M.D. Hirsch and H. Mahr, Chem. Phys. Letters 71 (1980) 27.

[5] L.A. Brey, G.B. Schuster and H.G. Drickamer, J. Chem. Phys. 67 (1976) 2648.

[6] E.P. Ippen, C.V. Shank and A. Bergman, Chem. Phys. Letters 38 (1976) 611 .

[7] G.S. Beddard, T. Doust and M.W. Windsor, in: Picosecond phenomena, Vol. 2, eds. R.M. Hochstrasser, W. Kaiser and C.V. Shank (Springer, Berlin, 1980) p. 167.

[8] D.A. Cremers and M.W. Windsor, Chem. Phys. Letters 71 (1980) 27.

[9] D.A. Cremers, Thesis, Washington State University (1980), unpublished.

[10] K.M. Keery and G.R. Fleming, Chem. Phys. Letters 93 (1982) 322. 
[11] V. Sundström, T. Gillbro and H. Bergström, Chem. Phys. 73 (1982) 439.

[12] V. Sundström and T. Gillbro, J. Chem. Phys. 81 (1984) 3463; Chem. Phys. Letters 110 (1984) 303.

[13] E. Ảkesson, H. Bergström, V. Sundström and T. Gillbro, Chem. Phys. Letters 126 (1986) 385.

[14] B. Bagchi, Intern. Rev, Phys. Chem. 6 (1987) 1.

[15] B. Bagchi, G.R. Fleming and D.W. Oxtoby, J. Chem. Phys. 78 (1983) 7375;

B. Bagchi, S. Singer and D.W. Oxtoby, Chem. Phys. Letters 99 (1983) 225.
[16] B. Bagchi, Chem. Phys. Letters 115 (1985) 209.

[17] B. Bagchi, Chem. Phys. Letters 128 (1986) 521.

[18] B. Bagchi, Chem. Phys. Letters 135 (1987) 553.

[19] D. Ben-Amotz and C.B. Harris, Chem. Phys. Letters 119 (1985) 305.

[20] G.R. Fleming, Chemical applications of ultrafast spectroscopy (Oxford Univ. Press, Oxford, 1986) ch. 6.

[21] R.W. Zwanzig and A. Harrison, J. Chem. Phys. 83 (1985) 5861.

[22] I. Artaki and J. Jones, J. Chem. Phys. 82 (1985) 3360.

[23] B. Bagchi, unpublished results. 